Borghi P., Cavalca G. (2015). Frontiere della rappresentanza: potenzialità e limiti organizzativi dell'offerta rivolta ai professionisti indipendenti in Sociologia del Lavoro, n.4 (140) Milano, Franco Angeli.

\title{
Frontiere della rappresentanza: potenzialità e limiti organizzativi dell'offerta rivolta ai professionisti indipendenti
}

L'eterogeneo mondo dei professionisti italiani è caratterizzato da un significativo e progressivo livello di segmentazione. I professionisti di cui parliamo, quelli appartenenti alle professioni liberali e quelli afferenti alle nuove professioni ${ }^{1}$ sperimentano condizioni di lavoro, possibilità di guadagno, opportunità lavorative e prospettive di carriera assai diversificate; questa eterogeneità si rispecchia nell'altrettanto variegato livello di soddisfazione per la propria carriera professionale.

Queste tendenze sono il risultato di una molteplicità di fattori quali un mercato del lavoro caratterizzato da una crescente flessibilità e un contesto produttivo nazionale a debole dinamicità, scarse misure di welfare, lunghi periodi di lavoro non pagato ad inizio carriera, uno scarso riconoscimento delle competenze professionali, un forte individualismo, endemico fra $\mathrm{i}$ professionisti, che ostacola la sedimentazione di identità collettive in grado di contrastare l'isolamento professionale e la carenza di rappresentanza.

Tutti questi aspetti sono stati accentuati dalla crisi economicofinanziaria internazionale che dal 2008 ha giustificato processi di ristrutturazione delle imprese, tagli alle consulenze, peggioramento delle condizioni di lavoro e delle aspettative di guadagno per molti professionisti. Questi fattori hanno condizionato i recenti tentativi di alcune organizzazioni, sia tradizionali che nuove, di definire un'offerta di rappresentanza rivolta ai professionisti indipendenti. In questo contributo si propone un'analisi comparativa di tre organizzazioni rilevanti, caratterizzate da differenti origini, strutture e culture organizzative: la CGIL, la Rete Redattori Precari e l'Associazione Consulenti del Terziario Avanzato. Lo studio utilizza i risultati di una recente esperienza di ricerca su organizzazioni di rappresentanza e professionisti indipendenti nell'area milanese e li integra con interviste mirate all'analisi delle strategie organizzative e con l'analisi dei documenti prodotti dalle organizzazioni.

\section{I professionisti indipendenti e le organizzazioni di rappresentanza}

\footnotetext{
${ }^{1} \mathrm{Si}$ intendono le professioni non ordiniste nel terziario avanzato.
} 
Sotto l'etichetta di professionisti indipendenti rientrano sia quelli che operano in ambito ordinistico sia quelli delle nuove professioni non ordiniste (es: artisti, creativi, informatici, operatori del benessere, consulenti, designer). Tutti condividono condizioni di lavoro mediamente peggiori di quelle sperimentate dalle generazioni precedenti, inoltre operano in una zona grigia che sta fra il lavoro subordinato e il lavoro autonomo (Barbieri, 1999). Per le nuove generazioni l'esperienza lavorativa appare meno promettente rispetto a quella dei colleghi più anziani, non solamente in termini di remunerazione, ma anche di prestigio sociale (Bellini, 2013). La deregolamentazione parziale e selettiva del mercato del lavoro europeo degli ultimi decenni (Esping-Andersen e Regini, 2000) ha relegato sempre di più $\mathrm{i}$ giovani nella condizione di outsiders e mid-siders (Madama et al., 2009). Questo generale peggioramento delle condizioni di lavoro continua avere significativi riflessi anche fra i professionisti indipendenti. Alcuni di questi sono "economicamente dipendenti" poiché operano principalmente o esclusivamente per un solo committente da cui ricevono stringenti indicazioni e tempistiche per la realizzazione del lavoro commissionato. A fronte di ciò, $\mathrm{i}$ diritti e le protezioni sociali, la rappresentanza collettiva e l'accesso al welfare, sono piuttosto limitati (Coletto e Pedersini, 2010; Berton et al., 2009). Sebbene questa condizione di debolezza sia ampiamente dibattuta, l'organizzazione collettiva dei professionisti indipendenti e la loro rappresentanza rimangono allo stesso tempo questioni ancora irrisolte e una sfida per i sindacati e le altre organizzazioni dei lavoratori (Cella, 2012). Se la fatica a rappresentare gli interessi dei lavoratori è strutturale (Accornero, 1992; Baglioni, 2008), essa si accentua ancora di più nel caso dei lavoratori non standard (Regalia, 2006) e delle nuove generazioni di professionisti indipendenti, spesso lontani dalla cultura organizzativa e dalle prassi della rappresentanza collettiva classica.

Secondo l'approccio del revitalisation of unionism (Frege e Kelly, 2004) le organizzazioni di rappresentanza affrontano queste difficoltà attraverso due strategie organizzative: l'organising, le iniziative di mobilitazione atte a costruire nuova appartenenza (membership) che fanno protendere l'organizzazione verso le relazioni con l'ambiente esterno o il servicing, la fornitura di servizi ai propri membri che tende a concentrare le risorse organizzative sulla formazione del personale e sulla strutturazione interna (Regalia, 2009; Beccalli et al., 2014).

A partire dalla metà degli anni '90, Milano (e in una certa misura l'intero territorio nazionale) ha visto una crescita e una differenziazione delle organizzazioni che si rivolgono ai professionisti indipendenti: 1) organizzazioni trasversali che si rivolgono a professionisti di diversi settori; 2) organizza- 
zioni che si rivolgono ai professionisti ordinisti; 3) organizzazioni che si rivolgono ai professionisti delle professioni neo-regolamentate ${ }^{2}$ (Mingione et al., 2014).

Al di là delle grandi tematiche comuni (lotta all'isolamento professionale ed erogazione di servizi), gli obiettivi generali dei tre gruppi di organizzazioni sono differenti: si va dalla rappresentanza classica fortemente strutturata dei sindacati, ad un'idea di rappresentanza reticolare, calibrata sui bisogni professionali, declinata su singoli settori (o trasversale) promossa dalle nuove organizzazioni. Queste differenze nascono sia dalle diverse culture organizzative, sia dalle scelte contingenti in un contesto relativamente nuovo in cui non si sono ancora consolidate strategie d'azione e coalizioni. Fin dall'entrata in vigore delle nuove tipologie di contratto flessibili i sindacati confederali hanno creato strutture interne specializzate sui lavoratori atipici, che per estensione si sono poi occupate anche dei nuovi professionisti. Nonostante questo sforzo e l'aumento dei lavoratori potenzialmente bisognosi di protezione, i sindacati non sono riusciti ad attirare un maggior numero di iscritti (Baglioni, 2008). Le nuove organizzazioni emergono quando si fa evidente questa scarsa capacità dei sindacati di rilevare e rappresentare i bisogni dei lavoratori non-standard.

\section{Gli aspetti organizzativi}

Sono tre gli elementi intorno ai quali si sviluppa l'analisi delle strategie organizzative: la struttura, la cultura e l'apprendimento.

Il panorama dell'offerta organizzativa può essere interpretato ricorrendo alla classica opposizione tra organizzazione razionale-burocratica e approccio organicista (Burns, Stalker, 1961). Da una parte l'organizzazione come macchina di Taylor e Fayol (Morgan, 2012), o razionale-burocratica di Weber, poi problematizzata tra gli altri da Merton, Crozier e Simon (Bonazzi, 2008), caratterizzata da forte strutturazione verticale, specializzazione del lavoro e professionalizzazione. Dall'altra l'organizzazione come sistema aperto (Morgan, 2012) e la struttura a rete (Butera, 1990): queste organizzazioni utilizzano come risorse l'orizzontalità, l'interazione con l'ambiente circostante, la collaborazione e l'interscambiabilità dei soggetti. Verso il primo polo troviamo le organizzazioni tradizionali, i sindacati e gli ordini professionali, verso il secondo le nuove organizzazioni costruite come reti

\footnotetext{
${ }^{2}$ L.4/2013, Disposizioni in materia di professioni non organizzate (GU n.22 del 26-12013)
} 
auto-organizzate.

Le nuove organizzazioni riservano grande importanza al loro campo organizzativo (DiMaggio e Powell, 1983) e alla relazione con i rappresentati, ma spesso scontano la mancanza di strutture stabili e di personale stipendiato che possa dedicare tempo, non volontario e aggiuntivo al lavoro individuale, alle attività di organising e di servicing (Regalia, 2009). Ad una maggiore democraticità e capacità di relazione con i professionisti fanno da contraltare le maggiori difficoltà nell'incrementare la membership.

La cultura organizzativa rappresenta una risorsa fondamentale per l'integrazione organizzativa (Schein, 1985), nel caso della rappresentanza la membership, ma se non viene adattata ai cambiamenti rischia di trasformarsi in "prigione psichica" (Morgan, 2012). Le organizzazioni si costruiscono intorno alle rappresentazioni che loro stesse hanno della realtà: la loro interpretazione dell'identità dei professionisti, dei loro bisogni e degli obiettivi da raggiungere per migliorarne le condizioni di lavoro e di vita, definisce soggettivamente il modello di rappresentanza e le routine organizzative che mettono in campo (Weick 1997). Nei periodi di crisi e mutamento sociale le organizzazioni necessitano di adattare la loro cultura per non replicare modelli di azione e di struttura incapaci di rispondere ai bisogni e alle identità nuove dei soggetti da rappresentare.

L'apprendimento organizzativo (Argyris e Schön, 1978) risulta quindi essenziale come risorsa di adattamento e di efficacia della rappresentanza; in linea di principio più un'organizzazione è complessa e strutturata verticalmente minore è la sua capacità di apprendere e di farlo in tempi rapidi.

\section{I tre casi studio: ReRePre, ACTA e la Consulta delle Professioni}

\subsection{Rete dei Redattori Precari}

ReRePre $^{3}$ è stata creata nel 2008 da redattori, traduttori, correttori di bozze ed altre figure professionali impegnate nel settore editoriale, che storicamente ha un ruolo importante nell'economia dell'area milanese ma si distingue anche per un crescente deterioramento delle condizioni di lavoro in corrispondenza con un prolungato periodo di sofferenza dell'intero settore confermata anche dagli ultimi dati disponibili ${ }^{4}$.

\footnotetext{
${ }^{3}$ Per una trattazione diffusa del caso studio si rimanda a Zambelli 2012.

${ }^{4}$ Gli unici segnali positivi sono circoscritti alla crescita del mercato digitale ed alla
} 
I principali nodi della rete si formano a Milano, Roma e Bologna e svolgono un importante ruolo nel dare voce ai lavoratori precari dell'editoria. Attraverso un'intensa attività on-line molti lavoratori, spesso isolati, hanno la possibilità di unirsi ad una comunità che discute la propria condizione lavorativa e cerca di trovare soluzioni collettive per contrastare i processi di domestication (Bologna e Fumagalli, 1997) e la precarietà strutturale del settore, che ha importanti ed altrettanto strutturali ricadute sulle traiettorie di mobilità sociale e sulle scelte di vita.

Se la rete ReRePre nasce quindi da un bisogno a cui le organizzazioni di rappresentanza, sindacati in primo luogo, non sono riusciti a dare risposta sia per questioni legate alla loro cultura organizzativa sia per vincoli oggettivi determinati dai dispositivi contrattuali, va però rilevato come specifiche e favorevoli condizioni locali, intrecciate all'iniziativa di singoli individui interni alle organizzazioni, generano inattese collaborazioni fra organizzazioni, come nel caso della prima indagine nazionale sul lavoro precario nell'editoria (Dieci et al., 2013), promosso dal SILC-CGIL di Milano e Bologna e IRES Emilia Romagna, in collaborazione proprio con ReRePre e il Sindacato Traduttori Editoriali. È questo il primo tentativo di descrivere in modo sistematico il ricorso strutturale al lavoro precario nel settore dell'editoria ed allo stesso tempo il primo segnale importante della volontà di produrre una conoscenza completa e condivisa su questi temi, condizione indispensabile per strutturare un dibattito sui bisogni di rappresentanza di questa tipologia di lavoratori.

I cambiamenti strutturali nel settore dell'editoria hanno accentuato i processi di esternalizzazione iniziati anni prima, che in questa fase arrivano a pieno compimento: se per anni i lavoratori con contratto a progetto si sono visti rinnovare pressoché automaticamente il contratto annuale, negli ultimi anni, e in particolare dopo la riforma Fornero, anche questo automatismo viene messo in discussione. Parte delle attività strategiche vengono appaltate all'esterno a singoli professionisti con partita IVA o a piccole agenzie sovente create da persone che prima lavoravano all'interno delle case editrici. Questo quadro di precarietà è completato da un costante ricorso a lunghi periodi di pratica non pagata o sottopagata per chi è in fase d'ingresso lavorativo.

ReRePre svolge ruolo strategico: nata per rispondere a bisogni ignorati dalle principali organizzazioni di rappresentanza, ha giocato un ruolo fondamentale nel renderli elemento di dibattito pubblico, stimolando una sep-

vendita dei titoli di editori italiani all'estero $(+7,3 \%$ nel 2013$)$; i rimanenti dati di scenario indicano una riduzione del bacino di lettori $(-4,7 \%)$, dei titoli pubblicati $(-4,1 \%)$, delle copie vendute $(-2,3 \%)$ e del fatturato $(-6,8 \%)$ (AIE, 2014). 
pur lenta e parziale reazione di parte dei sindacati. Inoltre l'investimento nella nuove forme di comunicazione web-based (siti internet e social media) ha favorito la creazione di una micro-comunità, favorendo in questo modo il rafforzamento di un'identità professionale dei lavoratori.

Dall'analisi di questo caso studio emergono punti sia di forza sia di debolezza nelle strategie organizzative. L'orizzontalità che caratterizza la struttura organizzativa è funzionale e coerente rispetto ai mezzi di comunicazione e di azione che partono spesso da internet e dai social network. Se tali strumenti risultano strategici per stimolare in modo virale un dibattito pubblico, non garantiscono però l'attivazione di pratiche di protesta e di rappresentanza fuori dal web. Queste sono ostacolate da diversi fattori: le condizioni di lavoro (domestication, precarietà, controllo sociale esercitato dai datori di lavoro presenti e potenziali e conseguente ricattabilità), l'individualismo dei lavoratori stessi (convinzione di poter fare da sé, fiducia nelle proprie reti sociali per una continuità lavorativa, speranza di riuscire a ottenere miglioramenti contrattuali con i committenti), l'impossibilità di dedicare risorse ad attività che non siano il lavoro e la gestione familiare. Questi elementi producono la paradossale situazione in cui una discussione pubblica sull'insostenibile condizione dei precari dell'editoria senza eclatanti e partecipate azioni di protesta nei posti di lavoro:

«Prima dell'indagine "Editoria Invisibile" promossa da ReRePre e CGIL nessuno sapeva quanti fossero i precari nell'editoria. Questa è la ragione per cui il primo obiettivo importante era far sapere ai lavoratori precari che la loro condizione era la stessa di tantissimi altri colleghi. Avevamo bisogno che si diffondesse la consapevolezza di questa condizione comune ma questo significava anche che qualcuno doveva esporsi pubblicamente e personalmente, qualcuno doveva metterci la faccia. (...) Quando la grande impresa per cui lavoravo non $\mathrm{mi}$ ha rinnovato il contratto, mi è stato detto espressamente che io conoscevo molto bene i miei diritti e che avevano seguito tutti gli eventi pubblici in cui avevo parlato delle nostre condizioni di lavoro» (Int. attivista ReRePre)

Un secondo e più strutturale elemento di debolezza organizzativa di ReRePre, comune a tutte le organizzazioni orizzontali e informali, è la natura volontaria e non retribuita del lavoro degli attivisti. Le attività della Rete sono rese possibili da una forte motivazione degli attivisti che impegnano il loro tempo libero per garantire una certa continuità. Sul medio periodo questa condizione però impedisce di definire ed articolare strategie che possano generare attività di rappresentanza strutturate, privilegiando così l'orga- 
nizzazione di singoli eventi, la cui visibilità può essere elevata, temporalmente limitata, senza però tradursi automaticamente in strategie più complesse.

La natura informale della rete genera, inoltre, una parziale mancanza di legittimazione istituzionale necessaria per un'interlocuzione stabile con i decisori politici. Infine l'attività dell'organizzazione può subire più facilmente battute di arresto sia per difficoltà legate al ricambio generazionale sia per ragioni contingenti legate alle traiettorie personali degli attivisti che la sostengono.

\subsection{ACTA}

L'Associazione dei Consulenti del Terziario Avanzato è una delle più innovative associazioni professionali italiane; riunisce i freelance che lavorano come formatori, tecnici informatici, consulenti, progettisti, traduttori con clienti privati e pubblici. L'associazione ha quattro sedi regionali: Lombardia, Piemonte, Toscana e Lazio, che gestiscono autonomamente l'attività sul territorio. ACTA è parte dello European Forum of Independent Professionals (EFIP) che svolge un'intensa attività di lobby a livello europeo. È stata fondata a Milano nel 2004 da un gruppo eterogeneo di professionisti non collegati a sindacati e partiti politici, quando la rappresentanza dei professionisti stava diventando un tema emergente anche a causa dei significativi cambiamenti nel sistema produttivo e nel mercato del lavoro. In quegli anni le organizzazioni professionali esistenti e ancor più i sindacati scontavano un forte ritardo nel riconoscere e interpretare tali cambiamenti strutturali e la creazione di ACTA è stato uno dei segnali di reazione a questo vuoto di rappresentanza.

La proposta di rappresentanza di ACTA è trasversale alle professioni, si rivolge a un blocco sociale di liberi professionisti impegnati in diversi settori produttivi con caratteristiche e bisogni simili; tali somiglianze sono più significative di quelle che possono accomunare liberi professionisti e dipendenti nello stesso settore (Int. Cofondatore 1 ACTA). L'intento è di superare una prospettiva corporativa per aggregare soggetti che possano agire collettivamente per costruire prospettive comuni. Nel contesto italiano questo approccio risulta innovativo rispetto a quello proposto fino ad anni recenti da associazioni professionali e sindacali.

Le campagne di sensibilizzazione promosse da ACTA in materia di trattamento previdenziale, assistenziale e fiscale, sviluppate a partire da un'intensa comunicazione via web, hanno giocato un ruolo strategico nel 
conquistare una significativa visibilità mediatica, che ha poi generato opportunità ulteriori di pressione sui decisori istituzionali nel tentativo di negoziare migliori condizioni per i liberi professionisti.

La struttura organizzativa si basa sul lavoro volontario dei soci, che garantiscono sia la gestione delle campagne di sensibilizzazione sia le attività di interlocuzione con i soggetti istituzionali e con il tessuto associativo che definisce il campo organizzativo (DiMaggio e Powell, 1983) in cui ACTA opera. Nei primi anni di vita dell'associazione è prevalsa una gestione orientata alla promozione ed alla visibilità nel tentativo di allargare la base associativa (rafforzamento della membership). Ben presto però l'associazione ha verificato le difficoltà, comuni a molti dei soggetti che operano in questo contesto, nell'acquisire nuovi soci e per questo motivo all'organising è stata affiancata, con gradualità e prudenza, l'attività di servicing riservata ai soci: consulenze in materia fiscale, legale e di gestione della comunicazione, momenti formativi ad hoc a prezzi ridotti, stipula di convenzioni per pensioni integrative e assicurazioni sanitarie.

ACTA condensa molte delle potenzialità e delle contraddizioni della rappresentanza dei professionisti indipendenti. È riuscita ad accumulare in poco più di un decennio una notevole reputazione: la visibilità generata dalle campagne on-line ha avuto una ricaduta positiva in primo luogo sui media tradizionali e anche sulla legittimazione istituzionale. Questa capacità si scontra però con la difficoltà endemica a trasformare la partecipazione sporadica on-line in partecipazione continuativa e in presenza: un caso significativo e tutt'altro che contingente di conclamata capacità di "rappresentanza a rappresentatività intermittente". Tale situazione ha varie spiegazioni, molte delle quali connesse ai soggetti da rappresentare, per loro natura mobili, frammentati e isolati o, in alcuni casi, corporativi. La via del servicing può essere interpretata come un tentativo di fornire ai propri soci vantaggi concreti che monetizzino il pagamento della quota d'iscrizione, pur nella consapevolezza che il valore aggiunto rimane la possibilità di essere un interlocutore autorevole dei decisori istituzionali. La scelta del servicing può però provocare tensioni organizzative: mantenere un'offerta stabile di servizi significa, in una certa misura, andare verso la professionalizzazione e la verticalizzazione della struttura, introducendo criteri di gestione imprenditoriale e ridiscutendo sia l'orizzontalità organizzativa sia la base volontaria delle attività associative.

Risulta molto interessante il posizionamento di ACTA in merito ad uno dei cambiamenti più significativi nella giurisprudenza sulle professioni: la legge $4 / 2013$, primo tentativo di creare una cornice giuridica per il gran numero di nuove professioni sorte nel sistema post-industriale e risultato di 
una forte attività di lobby promossa da un gruppo di associazioni professionali che si riconoscono in associazioni di secondo livello come COLAP, Confprofessioni, CNA.

ACTA è stata molto critica rispetto alle potenziali ricadute di questa legge sulla possibilità di migliorare l'offerta di rappresentanza, poiché essa andrebbe a favorire gli interessi delle associazioni in tema di formazione e di riconoscimento delle professionalità anziché sostenere i bisogni e gli interessi dei professionisti:

«Se le organizzazioni professionali sono coinvolte nella certificazione delle competenze professionali e nell'erogazione di un'offerta formativa, non saranno interessate ad affrontare i problemi reali di professionisti, come i mancati pagamenti, l'esclusione da misure di welfare, i regimi di tassazione [...] che sono le vere ragioni da cui partire per fornire un'offerta di rappresentanza.» (Int. Cofondatore 2 ACTA).

Allo stesso tempo però si riconosce l'errore strategico che, nella fase di approvazione della legge, ha ridotto le possibilità di influenzare il dibattito:

«Per molto tempo abbiamo interagito criticamente solo con i sindacati, mentre molte delle contraddizioni si trovano nelle organizzazioni professionali (...) Non abbiamo seguito da vicino il dibattito che ha portato alla nuova legge sulle professioni (...) Questo errore non ci ha permesso di prendere una posizione critica forte contro l'approvazione della legge sulle nuove professioni che è, a mio parere, il più grande ostacolo per la costruzione di una forte e strutturata offerta di rappresentanza per i professionisti». (Int. Cofondatore 2 ACTA)

La strategia di ACTA implica quindi un dibattito continuo e dialettico sia con i sindacati sia con le altre associazioni di categoria, nel tentativo di costruire alleanze nel contesto della rappresentanza dei professionisti indipendenti che non è sufficientemente strutturato per poter definire alleanze stabili e linee d'azione consolidate. Nell'attuale situazione prevalgono, quindi, l'esplorazione del campo organizzativo, il sostegno al dibattito pubblico sui professionisti, la sperimentazione di alleanze temporanee che generano, come effetto secondario ma non meno importante, autoriflessività e ridefinizione delle strategie d'azione.

\subsection{La Consulta delle Professioni della CGIL}


La Consulta delle Professioni della CGIL rappresenta uno dei tentativi più innovativi di dare rappresentanza ai professionisti. È una nuova struttura interna al sindacato, che si pone come ponte verso il mondo delle professioni, includendo organizzazioni di primo e secondo livello, così come singoli professionisti ed esperti. La CGIL in questo modo prova a superare il limite storico del sindacato, ben più grave in questa fase storica, di limitare la rappresentanza a impiegati e lavoratori dipendenti. La Consulta si ripromette di includere tutti i professionisti, non solo gli autonomi indipendenti, ma anche quelli dipendenti di aziende e pubbliche amministrazioni.

Un'operazione innovativa - probabilmente ancora non del tutto pienamente realizzata e accettata all'interno della CGIL - anche dal punto di vista organizzativo, che supera la visione classica dell'organizzazione burocratica per aprirsi all'ambiente circostante con una apposita struttura protesa alla costruzione di relazioni stabili interpretando a suo modo il modello di organizzazione a rete e di sistema aperto. Anche dal punto di vista della cultura organizzativa, inoltre, il salto è notevole, tanto che:

«le maggiori difficoltà alla nascita della Consulta (2009/2010) sono venute dall'interno della Cgil perché un atto così innovativo, come costruire un organismo della Cgil ma con all'interno associazioni e gruppi di professionisti esterni con i quali confrontarsi e concordare politiche ed azioni comuni, non era facile da accettare per chi pensava di detenere la rappresentanza di tutto il lavoro a prescindere dalla reale rappresentatività tra i lavoratori». (Int. responsabile Consulta)

Non si tratta del primo tentativo operato dalla CGIL (e dal sindacalismo italiano): fin dal secondo dopoguerra il maggiore sindacato ha organizzato alcune categorie di lavoratori autonomi (edicolanti, scrittori, attori, tassisti) (Imola 2011: 283-290). Lo stesso Nidil (Nuove Identità di Lavoro), nato nel 1998 per rappresentare i lavoratori atipici, ha poi tentato con molta difficoltà di mettersi in relazione anche con i professionisti indipendenti (Mingione et al., 2014).

La Consulta delle Professioni viene lanciata all'inizio del 2010 dopo un primo anno di colloqui e confronti tra CGIL, ordini e associazioni professionali ed esperti, come strumento istituzionale che raccoglie $\mathrm{i}$ bisogni delle professioni e dei professionisti e propone riforme ai policy makers. Al momento dell'indagine essa includeva circa 60 associazioni e gruppi professionali, diverse strutture della CGIL, singoli professionisti in 
qualche modo rappresentativi di specifici settori professionali e, infine, esperti e studiosi (Int. responsabile Consulta)

La strategia della CGIL punta esplicitamente a fornire un'unica forma di rappresentanza ai lavoratori al di là della condizione contrattuale, includendo quindi anche i lavoratori più qualificati degli stessi settori lavorativi e in un certo senso trasversalmente alle categorie.

A questo fine al centro dell'azione di rappresentanza rimane il contratto nazionale come strumento di trattativa, allargando però la platea ai professionisti e a tutte le forme di lavoro non subordinato. Il CCNL necessita infatti di essere adeguato e rinnovato come strumento per includere il lavoro autonomo, in particolare la sua parte più debole e per creare un nuovo sistema di contrattazione "inclusiva":

«... deve affrontare con forza e convinzione il tema della regolazione contrattuale senza la quale non si esercita nessuna rappresentanza perché viene meno il principio fondante del Sindacato che è quello di unire i lavoratori, tutti i lavoratori, per conquistare condizioni di lavoro e di vita migliori per tutti» (Int. responsabile Consulta)

La strategia è doppia (Imola 2011: 55-66): da una parte la nuova struttura multi-organizzativa della Consulta che elabora e promuove iniziative legislative cercando di rispondere ai bisogni dei professionisti; dall'altra le Federazioni, strutture organizzative tradizionali, assolvono al compito storico di contrattazione, includendo i professionisti dipendenti e indipendenti che lavorano nei singoli settori economici.

La partecipazione diretta ai meeting nazionali risulta piuttosto alta ma ovviamente $i$ professionisti, sia individuali sia rappresentanti di associazioni, hanno difficoltà a dedicarvi tempo, non potendo fare affidamento su rimborsi e permessi sindacali. Per questo la CGIL sta proponendo strutture regionali e metropolitane. Anche le regole di partecipazione sono minime per favorire l'apertura verso l'esterno. (Int. responsabile Consulta)

La Consulta delle Professioni sembra evidenziare la direzione della CGIL verso la conservazione del tradizionale ruolo di sindacato, aprendo però allo stesso tempo al cambiamento sia dell'obiettivo della rappresentanza, includendo i professionisti autonomi, sia delle relazioni con le altre organizzazioni, tessendo rapporti con gli attori collettivi che li rappresentano. La CGIL non rinuncia al ruolo di protezione dei lavoratori all'interno delle relazioni industriali, pur avendo ormai da tempo agito anche verso la protezione dei soggetti nel mercato del lavoro attraverso la 
partecipazione al sistema della formazione professionale e a quello pensionistico. Così come molti altri sindacati a livello europeo, la CGIL cerca, quindi, di far convivere cambiamenti organizzativi e strategici di tipo adattivo con la riaffermazione del suo ruolo di attore collettivo che si rivolge ai lavoratori che si riconoscono in un'identità collettiva (Pedersini, 2011), che anzi la Consulta vorrebbe (e forse potrà) contribuire a costruire. Da questo punto di vista la Consulta rappresenta una delle più interessanti innovazioni nel segno della revitalisation of unionism.

Nella sua strategia egemonica la CGIL gode ovviamente di un vantaggio di partenza, costituito dal ruolo storico di sindacato più rappresentativo, della dimensione organizzativa nazionale e della ancor consistente legittimazione sociale e istituzionale, che le permette di essere attraente per altre organizzazioni, le associazioni dei professionisti, che hanno da parte loro limitare possibilità di incidere sulle politiche:

«... la Cgil aggiunge alle proprie proposte su fisco, previdenza, ammortizzatori, tutele sociali ecc. una visione ma anche una possibilità d'azione, con la contrattazione collettiva, che nessuna associazione professionale è in grado d'agire da sola». (Int. responsabile Consulta)

La strategia di rappresentanza della CGIL punta a costruire relazioni con il più ampio numero di associazioni di professionisti e a favorire la collaborazione tra queste associazioni, solitamente molto legate alle singole identità professionali, e le Federazioni sindacali che attraverso la contrattazione collettiva possono affrontare sia le questioni specifiche (di un settore economico o professione) sia quelle generali e trasversali alle professioni, come per esempio l'equa remunerazione, le forme di contratto, l'allargamento delle coperture del welfare o la tassazione. In questo senso la Consulta sta collaborando intensamente anche con le associazioni di secondo livello che possono contribuire al rafforzamento della coalizione e alla condivisione di obiettivi. Le relazioni con le altre organizzazioni giocano un ruolo decisivo anche per aumentare la legittimazione della CGIL, attraverso la Consulta: più associazioni di professionisti, più settori e più professionisti individuali raccoglie, più crescono la capacità attrattiva come organizzazione di rappresentanza e il peso come interlocutore istituzionale.

\section{Conclusioni}


I tre casi studio, diversi per cultura politica e organizzativa, obiettivi e strategie, permettono di far emergere alcuni dei tratti caratteristici dell'offerta di rappresentanza rivolta ai professionisti indipendenti italiani e di comprenderne al contempo le origini e l'apporto di innovazione.

Una prima fase del processo di rappresentanza ha inizio quando dal basso nasce l'esigenza di rendere pubblica, attraverso un paziente lavoro di raccolta delle istanze e dei bisogni, una condizione di difficoltà comune a molti ma totalmente ignorata dal dibattito pubblico.

Il caso di ACTA e quello di ReRePre dimostrano come questo passaggio avvenga grazie a nuove organizzazioni che nascono per coprire un'area non efficacemente presidiata dai soggetti preesistenti. Sono i lavoratori precari dell'editoria e i consulenti del terziario che decidono di unirsi, denunciare la propria condizione e pretendere che interlocutori qualificati, sia sul fronte istituzionale sia sul fronte della committenza, prendano posizione. Uno degli strumenti privilegiati sono le campagne on-line, in grado di attrarre l'attenzione sia di iscritti e simpatizzanti sia dei mass media. La fase di attivazione passa, necessariamente e sempre di più, dalla rete e dai social media per poi tracimare nei mezzi di comunicazione tradizionali, una volta raggiunta una massa critica di adesioni e di discussioni sui temi scelti. I social media svolgono poi un ruolo centrale nel sostenere i processi di costruzione identitaria, laddove gli spazi di aggregazione tradizionali in ambito lavorativo si sono ridotti o sono del tutto assenti.

Diverso invece è il caso della Consulta delle Professioni che si connota come tentativo dall'elevato grado di innovatività in seno ad un'organizzazione fortemente strutturata e gerarchica come il sindacato, di recuperare il ritardo accumulato su questo fronte: è un processo di apprendimento organizzativo.

Possiamo dire che questo sia uno dei segnali del passaggio ad una seconda fase: dall'emersione dei problemi di uno specifico gruppo sociale, quello dei professionisti indipendenti e (spesso) precari attraverso la conquista di visibilità e identità nello spazio nell'arena mediatica (personale e di massa), alla coagulazione di proposte di rappresentanza che provano a strutturarsi intorno a coalizioni più composite, come appunto la Consulta. Questa fase è ancora caratterizzata dall'eterogeneità e dalla parzialità delle proposte di rappresentanza, nonché da un alto grado di conflittualità (anche interna), di collaborazione mirata a singole iniziative, alla sperimentazione di coalizioni più ampie di quelle rintracciabili nella fase precedente. È evidente infatti il divario di prospettive e di traiettorie che c'è fra i tre casi. Un'organizzazione orizzontale, autorganizzata e settoriale come ReRePre, 
in grado di proporre, negli anni, un importante lavoro di denuncia. Un'associazione come ACTA, anch'essa a struttura leggera e orizzontale, che si rivolge in modo trasversale ai liberi professionisti del terziario avanzato riuscendo in alcuni casi circoscritti ma importanti a influenzare le decisioni istituzionali. Infine, un'esperienza come la Consulta delle Professioni, nata dall'alto, che coinvolge in modo trasversale singoli professionisti, associazioni di professionisti ed associazioni di secondo livello, per costruire un tavolo di confronto nazionale che mira ad essere un soggetto autorevole ed egemone nell'interlocuzione con le istituzioni, sapendo di poter contare su una solida struttura organizzativa, quella del sindacato, che la promuove, ma dovendo gestire prospettive ed interessi articolati e non sempre coerenti al loro interno (quelli delle organizzazioni che partecipano alla Consulta, ivi inclusa la CGIL stessa).

In conclusione, dall'offerta di rappresentanza delle tre organizzazioni emergono precise dinamiche di cambiamento. Adottando la tipologia di Regalia (2009) sulle strategie di rappresentanza, basata su "interesse/disponibilità a innovare le logiche di azione" e "consapevolezza della portata del mutamento", le tre esperienze si collocano tutte nell'area dell'innovazione come diversificazione delle prassi della rappresentanza, mentre la vera e propria riconfigurazione della rappresentanza, la sfida più difficile, potrà avvenire a condizione che la sperimentazione della Consulta abbia davvero pieno appoggio nella CGIL, dando seguito al difficile tentativo di superare il dualismo insiders-outsiders, e che ACTA e ReRePre sappiano mantenere il ruolo innovativo e di sprone per il sindacato, ma anche se contribuire alla costruzione di un'identità collettiva non

corporativa tra i professionisti.

\section{Bibliografia}

Accornero A. (1992). La parabola del sindacato: ascesa e declino di una cultura, Bologna: il Mulino.

AIE (2014). Rapporto sullo stato dell'editoria. Roma: Associazione Italiana Editori.

Argyris, C., Schön, D. (1978). Organizational learning: a theory of action perspective. Reading, MA: Addison-Wesley.

Baglioni G. (2008). L'accerchiamento. Perché si riduce la tutela sindacale tradizionale. Bologna: il Mulino.

Barbieri P. (1999). Liberi di rischiare. Vecchi e nuovi lavoratori autonomi. Stato e mercato, 56, 2: 281-308.

Beccalli B., Meardi G. e Bacio M. (2014). Varietà delle forme di rappresentanza di cittadini e lavoratori: verso un'analisi internazionale e comparata. Quaderni di Rassegna Sindacale, XV, 1: 11-25. 
Bellini A. (2013), Gli avvocati e i paradossi della regolazione delle professioni. Un esercizio autoriflessivo. Paper presentato al Convegno nazionale AIS-ELO I fallimenti della regolazione, Bologna, 12-13 settembre.

Berton M., Ricchiardi S. e Sacchi S. (2009). Flex-insecurity. Perché in Italia la flessibilità diventa precarietà. Bologna: il Mulino.

Bologna S., Fumagalli A., a cura di (1997). Il lavoro autonomo di seconda generazione. Scenari del postfordismo in Italia. Milano: Feltrinelli.

Bonazzi G. (2008). Storia del pensiero organizzativo. Milano: Franco Angeli.

Burns T., Stalker G.M. (1961). The Management of Innovation. London: Tavistock.

Butera F. (1990). Il castello e la rete. Impresa, organizzazioni e professioni nell'Europa degli anni '90. Milano: Franco Angeli.

Cella G.P. (2012). Difficoltà crescenti per le relazioni industriali europee e italiane. Stato e mercato, 1: 29-54.

Coletto D., Pedersini R. (2009). Self-employed workers: industrial relations and working conditions. Dublin: Eurofound.

Dieci D., Fontani C. e Rinaldini F. (2013). Editoria invisibile. Un'inchiesta sui lavoratori precari dell'editoria. Bologna: Ires.

DiMaggio P.J., Powell W. (1983). The iron cage revisited: Institutional Isomorphism and Collective Rationality in Organizational Fields. American Sociological Review, 48: 147-60.

Esping-Andersen G., Regini M. (2000). Why Deregulate Labour Markets? New York: Oxford University Press.

Frege C., Kelly J. a cura di (2004). Varieties of Unionism: Strategies for Union Revitalization in a Globalizing Economy. Oxford: University Press.

Imola D., a cura di (2011). I professionisti e il sindacato: tra scoperta e innovazione. L'azione della CGIL nel lavoro professionale e la costituzione della Consulta delle professioni. Roma: Ediesse.

Madama I., Jessoula M., Graziano P. (2009) Flessibilità e sicurezza, per chi? Sviluppi di policy e conseguenze nel mercato del lavoro italiano. Stato e mercato, 3: 387-420, doi: $10.1425 / 30842$

Mingione, E., Andreotti, A., Benassi, D., Borghi, P., Cavalca, G. and Fellini, I. (2014). Le organizzazioni sociali e i giovani professionisti nell'area milanese. Quaderni di Rassegna Sindacale, XV, 1: 123-140.

Morgan G. (2012). Images. Le metafore dell'organizzazione. Milano: Franco Angeli.

Pedersini R. (2011). Differenze e individualizzazione: quale ruolo per le relazioni industriali? In: Imola D., a cura di, I professionisti e il sindacato: tra scoperta e innovazione. L'azione della CGIL nel lavoro professionale e la costituzione della Consulta delle professioni. Roma: Ediesse.

Rapelli S. (2012). European I-Pros: A Study, Research Report. London: PCG.

Regalia I., a cura di (2006). Regulating new forms of employment. Local experiments and social innovations. Abingdon/New York: Routledge.

Regalia I. (2009). Quale rappresentanza? Dinamiche e prospettive del sindacato in Italia. Roma: Ediesse.

Schein E.(1985). Organizational Culture and Leadership. San Francisco: Jossey-Bass.

Weick K.E. (1997) Senso e significato nell’organizzazione. Alla ricerca delle ambiguità e delle contraddizioni nei processi organizzativi. Milano: Raffaello Cortina.

Zambelli, L. (2012) Dalla frammentazione alla rete, il caso studio della Rete dei Redattori Precari. Tesi di Laurea Magistrale, Università di Trento, a.a. 2011/2012. 